\title{
Random vector space approach applied in integrated navigation information fusion of UAVs
}

\author{
Rongjun $\mathrm{Mu}^{1}$, Yuntian $\mathrm{Li}^{1, \mathrm{a}}$ and Yongzhi Shan ${ }^{2}$ \\ ${ }^{1}$ School of Astronautics, Harbin Institute of Technology, Harbin 150000, China \\ ${ }^{2}$ Institute of Aerial Ammunition, China North Industrial Corporation, Harbin 150000, China
}

\begin{abstract}
Federated Kalman Filter (FKF), is the most widely used distributed data fusion algorithm. Whilst FKF required local systems of the same system model, which is difficult to satisfy in most circumstances. How to balance the estimation accuracy and the calculating load is an urgent problem needs to be solved. Random Vector Space treats state predictions and estimations of both local and global modules as RVS bases equally. Then the state optimal estimation can be denoted through the combination of these bases. Replacing the time-updating of global module in FKF with RVS approach draw a higher level of accuracy with the same calculating time. Simulation results indicate the position and velocity estimation accuracy of three axes are improved by $1.59 \%, 1.53 \%, 1.29 \%$ and $19.9 \%, 13.3 \%, 17.6 \%$, respectively.
\end{abstract}

Keywords: random vector space; optimal estimation; data fusion; integrated navigation.

\section{Introduction}

Nowadays, with the rapid development in most respects of the modern society (e.g. economy, science, engineering and so on), UAVs become increasingly popular in many areas. For example, intelligence collection, disaster monitory, military support and so far. As the critical part of the UAV GNC(Guidance-Navigation-Control) system, the accuracy of navigation devices is of great importance. Many different navigation systems, e.g. inertial navigation system (INS), synthetic aperture radar (SAR), GPS, are applied to UAVs' navigation. Due to the limitation of different navigation system, such as INS calculating error accumulated through time; SAR obtains less precise measurement during areas without distinguishing topographic feature; GPS has a limited performance in the environment with strong electromagnetic interference. These drawbacks lead to the emergence of integrated navigation system which combined the advantage of each navigation system to obtain the optimal navigation information 1.

For the sake of the optimal data fusion of integrated navigation system, Kalman Filter (KF) and its derivative forms are used to fuse date from different navigation systems. The two approached of KF are called Centralized Kalman Filter (CKF) and Distributed Kalman Filter (DKF) according to its structure 2. Although CKF has a simple structure but it also has some inherent defects like poor fault tolerant capability and heavy computation load. Due to these limitations, DKF draws increasingly attention in the last two decades 3. The most widely used DKF algorithm is the Federated Kalman Filter proposed by N. A. Carlson in 19884.

a Corresponding author : yuntianlee91@163.com

(C) 2016. The authors - Published by Atlantis Press 
Though FKF is suitable for integrated navigation fusion, it relies on the information-sharing principle. If the information-sharing principle is reset, it is optimal, otherwise it is sub-optimal. Whilst, reset-FKF commonly has more computational load and inaccurate information from one local system may contaminate other systems through the reset process.

In this paper, an INS/SAR/GPS integrated system is introduced first, and state values and measurement values used for data fusion are defined. Then the RVS approach and its optimal fusion theorem are interpreted and compared with the Standard Kalman Filter (SKF) to indicate the relationship between each other. On this basis, the RVS global optimal fusion algorithm is applied in the global fusion process and corresponding simulation is implemented. What comes at the end are conclusions.

\section{Integrated navigation system components}

Currently, the widespread used navigation technology in UAVs are inertial navigation system, satellites navigation system, terrain matching, Doppler positioning and so on 5. Among these navigation technologies, INS, SAR and GPS are the increasingly trend of positioning technology applied in UAVs due to their high performance in independence, precision and reliability6.The structure of INS/SAR/GPS integrated navigation system of UAVs is given in Figure 1.

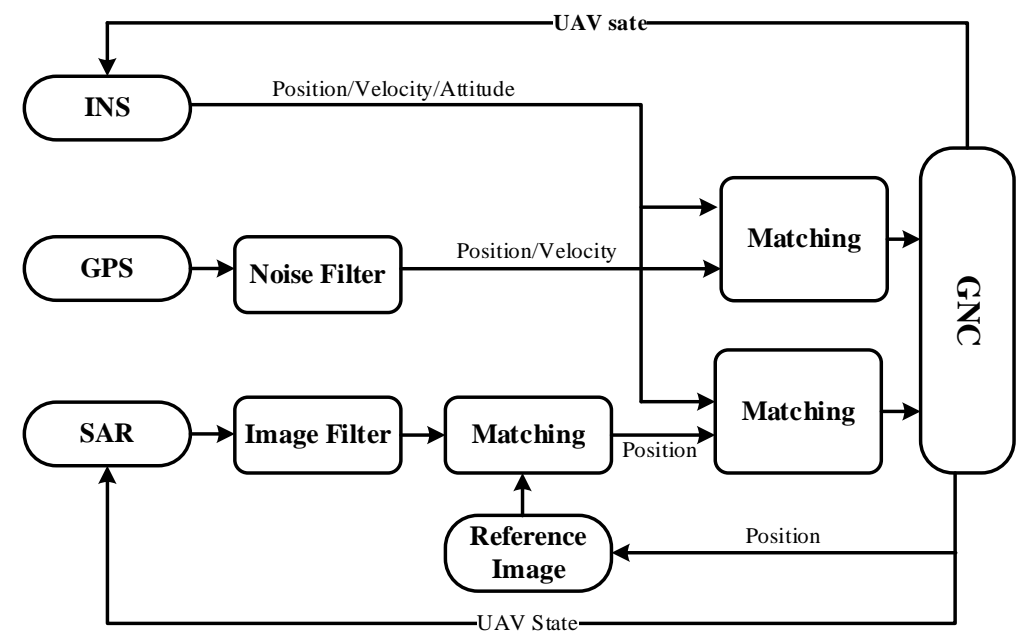

Figure 1. Structure of UAVs' INS/SAR/GPS Integrated Navigation System

Inertial Navigation System (INS) is a self-contained positioning technology which can predicate the vehicle's current statement (position, velocity and attitude) through its previous statement and inertial devices' measurement. The two major measurement information are the acceleration measured by accelerometers to obtain the velocity and position of vehicles, and the angular velocity sensed by gyroscopes to obtain the attitude angle.

The inertial navigation system updates its statement with various mechanization equations in different coordinate frames. The updating of the UAVs' statement is generally implementing in the geography coordinate (North-Up-East, NUE) as below 7:

$$
\left\{\begin{array}{l}
\dot{\mathbf{r}}^{n}=\mathbf{v}^{n}-\boldsymbol{\omega}_{e n}^{n} \times \mathbf{r}^{n} \\
\dot{\mathbf{v}}^{n}=\mathbf{C}_{b}^{n} f_{b}-\left(2 \boldsymbol{\omega}_{i e}^{n}+\boldsymbol{\omega}_{e n}^{n}\right) \times \mathbf{v}_{e}+\mathbf{g}_{1}^{n}
\end{array}\right.
$$

where the subscripts and superscripts 'i', e', 'n', 'b' represent the inertial reference coordinate (i-frame), the earth-centred fixed coordinate (e-frame), the navigation coordinate (n-frame) and the body coordinate (b-frame), respectively. In these equations, $\mathbf{r}^{n}$ and $\dot{\mathbf{r}}^{n}$ are position and position-rate 
vectors, respectively; $\mathbf{C}_{b}^{n}$ is the attitude matrix from b-frame to $\mathrm{n}$-frame; $f^{b}$ is the accelerometer specific force vector; $\boldsymbol{\omega}_{e n}^{n}$ and $\boldsymbol{\omega}_{i e}^{n}$ are $\mathrm{n}$-frame rotation velocity and earth rotation velocity, respectively. To avoid the singularity of euler angle updating approach, the quaternion approach is used to update the attitude of UAVs. The quaternion updating equations are:

$$
\left\{\begin{array}{l}
\dot{\mathbf{q}}_{b}^{n}=\frac{1}{2} \mathbf{q p}_{n b}^{b} \\
\mathbf{p}_{n b}^{b}=\left[0, \boldsymbol{\omega}_{n b}^{b T}\right]^{T}
\end{array}\right.
$$

where $\mathbf{q}$ and $\dot{\mathbf{q}}$ is the attitude quaternion and quaternion-rate, respectively; $\boldsymbol{\omega}_{n b}^{b}$ is the angular velocity sensed by gyroscope.

GPS positioning technology is the most widely used navigation aided technology no matter for civil or military use. In this system INS and GPS are integrated in loose combination. So the measurement of GPS are position and velocity, system measurements are given as:

$$
\mathbf{Z}_{g}=\left[\begin{array}{l}
\delta P_{N} \\
\delta P_{U} \\
\delta P_{E} \\
\delta V_{N} \\
\delta V_{U} \\
\delta V_{E}
\end{array}\right]=\left[\begin{array}{ll}
P_{N}^{I N S} & P_{N}^{G P S} \\
P_{U}^{I N S} & P_{U}^{G P S} \\
P_{E}^{I N S} & P_{E}^{G P S} \\
V_{N}^{I N S} & V_{N}^{G P S} \\
V_{U}^{I N S} & V_{U}^{G P S} \\
V_{E}^{I N S} & V_{E}^{G P S}
\end{array}\right]
$$

Emerging in 1960s, SAR is a positioning technology with high resolution and accuracy through image matching. SAR positioning procedure is given in Figure 2. SAR measurement can be used to correct the errors of INS and INS state can be used to compensate SAR, the measurements of SAR are given as:

$$
\mathbf{Z}_{S}=\left[\delta P_{N}, \delta P_{E}\right]^{T}
$$

where $\delta P_{N}$ and $\delta P_{E}$ is the north and east positioning error, respectively.

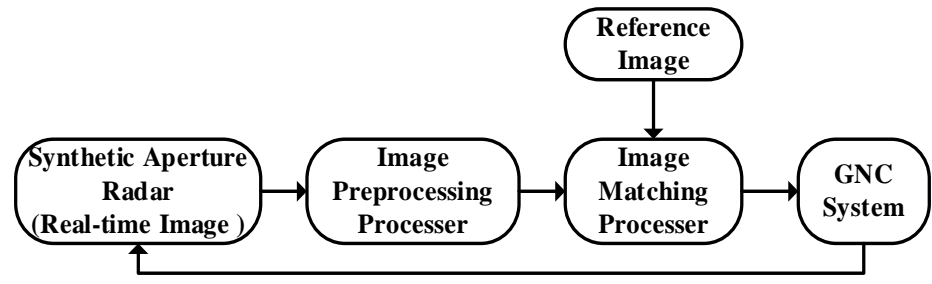

Figure 2. SAR Position Procedure

\section{Multi-sensor navigation data fusion algorithm}

\subsection{General statement of navigation data fusion}

The structure of INS/GPS/SAR integrated navigation data fusion system is given in Figure 3. At first, INS integrates with GPS and SAR, respectively. Then local predictions and estimations are transmitted to the global filter to obtain the global optimal estimation. 
In an integrated navigation system consist of various navigation subsystems, the most common distributed fusion algorithm is the Federated Kalman Filter (FKF)9. The FKF transfer the estimation and covariance between global filter and local filters through the so-called information-sharing factor principle[10].FKF acquires the optimal estimation by information-sharing factors and these factors are often scalar. RVS approach can modified this method by denoting the optimal estimation with a set of vector bases.

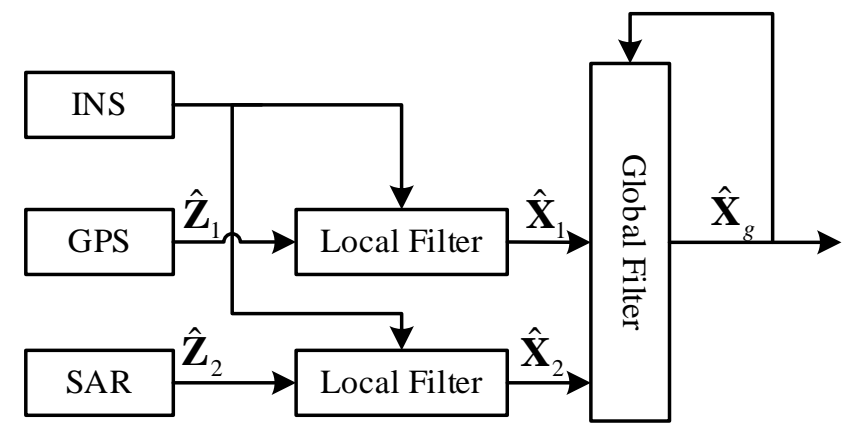

Figure 3. Structure of INS/GPS/SAR Data Fusion System

\subsection{Random vector space optimal fusion approach}

According to the optimal fusion theorem proposed by Yong Li in[11], if $\mathbf{r}_{i}(i=1,2 \cdots n)$ is a series of unbiased orthogonal vectors construct a random vector space(RVS) $\boldsymbol{\Theta}$, then any vector belongs to this space can be represented as:

$$
\mathbf{R}=\sum_{i=1}^{n} \boldsymbol{\alpha}_{i} \mathbf{r}_{i}
$$

where $\boldsymbol{\alpha}_{i}$ are the projection of $\mathbf{R}$ on $\mathbf{r}_{i}$ in random vector space $\boldsymbol{\Theta}$.

Denoting $\mathbf{S}_{i}$ as the mapping vector between global estimation $\hat{\mathbf{X}}_{g}$ and local estimation $\hat{\mathbf{X}}_{i}, \mathbf{P}_{i}$ as the covariance matrix of $\hat{\mathbf{X}}_{i}$, which means:

$$
\hat{\mathbf{X}}_{g}=\mathbf{S}_{i} \hat{\mathbf{X}}_{i} \quad \mathbf{P}_{i}=E\left[\hat{\mathbf{X}} \hat{\mathbf{X}}_{i}^{T}\right]
$$

Assuming $\hat{\mathbf{X}}_{g}$ can be denoted as a linear combination of $\hat{\mathbf{X}}_{i}$ :

$$
\hat{\mathbf{X}}_{g}=\sum_{i=1}^{n} \mathbf{K}_{i} \hat{\mathbf{X}}_{i}
$$

where $\mathbf{K}_{i}$ is the optimal coefficient matrix. Combined Eq.(6) and Eq.(7), we can obtain:

$$
\hat{\mathbf{X}}_{g}=\sum_{i=1}^{n} \mathbf{K}_{i} \mathbf{S}_{i}^{-1} \hat{\mathbf{X}}_{g} \Rightarrow \sum_{i=1}^{n} \mathbf{K}_{i} \mathbf{S}_{i}^{-1}=\mathbf{I}
$$

According to the optimal fusion principle, the smaller the trace of covariance matrix, the higher the accuracy of estimation[12]. So the optimal estimation problem can be translated into a optimization problem under the constriction Eq.(8), and corresponding Lagrange Function is: 


$$
F_{l}=\operatorname{tr}\left[\sum_{i=1}^{n} \mathbf{K}_{i} \hat{\mathbf{P}}_{i} \mathbf{K}_{i}^{T}+\boldsymbol{\Gamma}\left(\sum_{i=1}^{n} \mathbf{K}_{i} \mathbf{S}_{i}^{-1}-\mathbf{I}\right)\right]
$$

To minimize Eq.(9), $\mathbf{K}_{i}$ should satisfy $\frac{\partial F_{l}}{\partial \mathbf{K}_{i}}=0$. If $\sum_{i=1}^{n}\left(\mathbf{S}_{i}^{T} \mathbf{P}_{i}^{-1} \hat{\mathbf{X}}_{i}\right)$ is not a singular matrix, then we can obtain the optimal estimate of $\hat{\mathbf{X}}_{g}$ as:

$$
\left\{\begin{array}{l}
\hat{\mathbf{P}}=\left(\sum_{i=1}^{n} \mathbf{S}_{i}^{T} \mathbf{P}_{i}^{-1} \mathbf{S}_{i}\right)^{-1} \\
\hat{\mathbf{X}}_{g}=\mathbf{P}\left(\sum_{i=1}^{n} \mathbf{S}_{i}^{T} \mathbf{P}_{i}^{-1} \mathbf{X}_{i}\right)
\end{array}\right.
$$

As vectors corresponding to the dynamic system can be divided into three categories as prediction, estimation and observation. All of those vectors above can be used to construct the bases vectors in RVS by orthogonalization theorem [13]. And the global optimal data fusion algorithm through RVS are given below [14]:

$$
\begin{gathered}
\boldsymbol{\alpha}=\left[\mathbf{I}-\boldsymbol{\Phi} \hat{\mathbf{P}}(-1) \sum_{i=1}^{n} \mathbf{S}_{i}^{T} \boldsymbol{\Phi}_{i}^{T} \tilde{\mathbf{P}}_{i}^{-1} \mathbf{S}_{i}\right]^{-1} \\
\boldsymbol{\alpha}_{i}=-\boldsymbol{\alpha} \boldsymbol{\Phi} \hat{\mathbf{P}}(-1) \mathbf{S}_{i}^{T} \boldsymbol{\Phi}_{i}^{T} \tilde{\mathbf{P}}_{i}^{-1} \\
\overline{\mathbf{P}}_{i}=\boldsymbol{\Phi} \hat{\mathbf{P}}(-1) \mathbf{S}_{i}^{T} \boldsymbol{\Phi}_{i}^{T} \tilde{\mathbf{P}}_{i}^{-1} \hat{\mathbf{P}}_{i} \\
\overline{\mathbf{X}}=\mathbf{K} \tilde{\mathbf{X}}+\sum_{\mathbf{i}=\mathbf{1}}^{\mathbf{n}} \mathbf{K}_{\mathbf{i}} \hat{\mathbf{X}}_{\mathbf{i}} \\
\overline{\mathbf{P}}=\mathbf{K} \tilde{\mathbf{P}} \mathbf{K}^{T}+\sum_{i=1}^{n} \mathbf{K} \hat{\mathbf{P}}_{i} \mathbf{K}_{i}^{T}+\sum_{i=1}^{n} \mathbf{K} \overline{\mathbf{P}}_{i} \mathbf{K}_{i}^{T}+\sum_{i=1}^{n} \mathbf{K}_{i} \overline{\mathbf{P}}_{i}^{T} \mathbf{K}^{T} \\
\hat{\mathbf{P}}=\left(\overline{\mathbf{P}}^{-1}+\sum_{i=1}^{n} \mathbf{S}_{i}^{T} \hat{\mathbf{P}}_{i}^{-1} \mathbf{S}_{i}\right)^{-1} \\
\hat{\mathbf{X}}=\hat{\mathbf{P}}\left(\overline{\mathbf{P}}^{-1} \overline{\mathbf{X}}+\sum_{i=1}^{n} \mathbf{S}_{i}^{T} \hat{\mathbf{P}}_{i}^{-1} \hat{\mathbf{X}}_{i}\right)
\end{gathered}
$$

Due to the further use of system information, it can be inferred that the RVS data fusion algorithm must have the higher level accuracy than the FKF.

\section{Mathematical simulation}

In order to evaluating the estimation precision, the RVS algorithm, a simulation is implemented under Simulink Modules with a series of UAV flight data (trajectory and navigation data) gathering from particular unit.

Simulation time is from 1700 s to 2000 s, filtering period and simulation step are both 0.1 s, 
parameters of different sensors are listed in Table 1. Estimating the position and velocity of the UAV through FKF and RVS, respectively and simulation results are summarized in Figure 4, Figure 5 and Table 2.

Table 1. Parameters of different sensors

\begin{tabular}{c|c|c}
\hline & Accelerometers & Gyroscopes \\
\hline Bias & $50 \mu \mathrm{g}$ & $1^{\circ} / \mathrm{h}$ \\
\hline Random Noise & $5 \mu \mathrm{g}$ & $0.1^{\circ} / \mathrm{h}$ \\
\hline & SAR & GPS \\
\hline Positioning Error & $15 \mathrm{~m}$ & $10 \mathrm{~m}$ \\
\hline Velocity Error & None Measurement & $0.5 \mathrm{~m} / \mathrm{s}$ \\
\hline
\end{tabular}
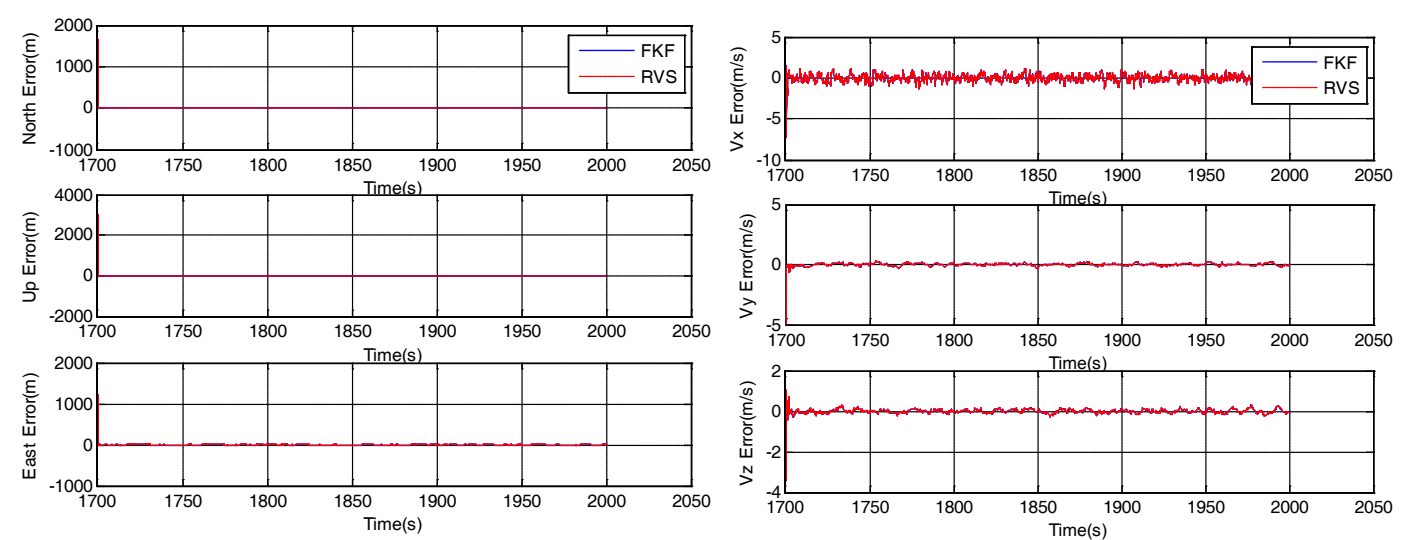

Figure 4. Estimation Precision of FKF\&RVS
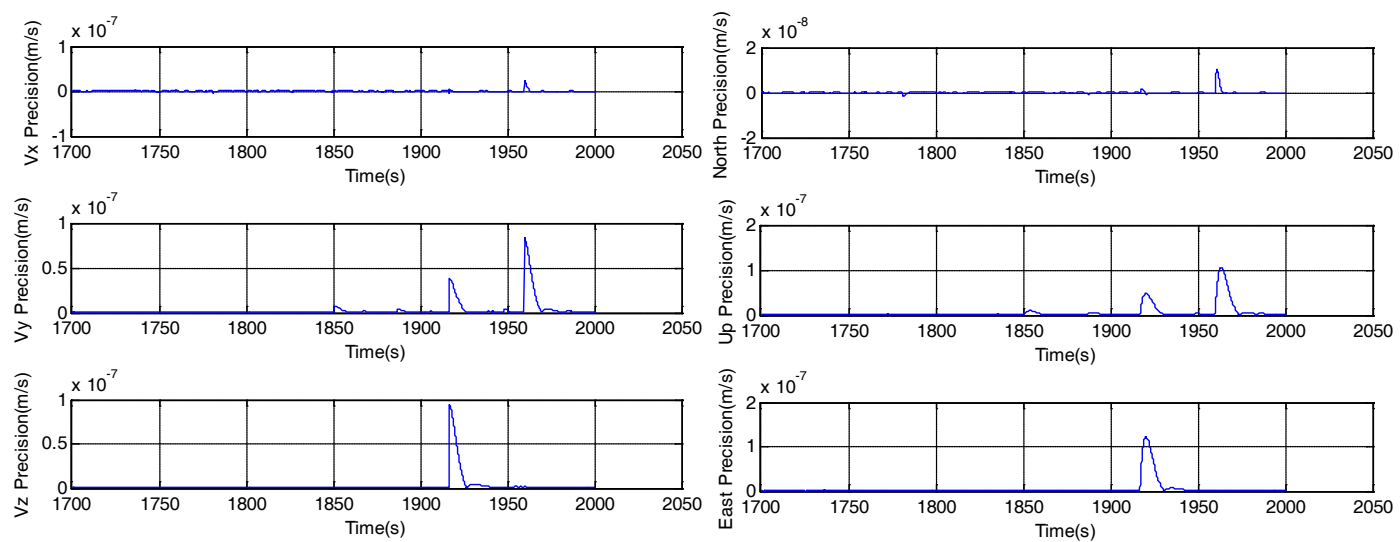

Figure 5. Precision Comparison of FKF\&RVS

Table 2. Precision Comparison of FKF\&RVS

\begin{tabular}{c|c|c|c|c}
\hline & & FKF & RVS & Comparison \\
\hline \multirow{3}{*}{ Position(m) } & North & 0.63 & 0.62 & 0.01 \\
\cline { 2 - 5 } & Up & 1.30 & 1.28 & 0.02 \\
\cline { 2 - 5 } & East & 0.77 & 0.76 & 0.01 \\
\hline \multirow{3}{*}{ Velocity(m/s) } & North & 0.020 & 0.016 & 0.004 \\
\cline { 2 - 5 } & Up & 0.015 & 0.013 & 0.002 \\
\cline { 2 - 5 } & East & 0.017 & 0.014 & 0.003 \\
\hline Calculating Time(s) & & 3.52 & 3.56 & 0.04 \\
\hline
\end{tabular}


Referring to the simulation results, we can see that calculating time of both algorithms are familiar (RVS costs $0.04 \mathrm{~s}$ more). Whereas, the performance of RVS is better than FKF. The position estimation precision of three axes are about 1.59\%,1.53\%,1.29\% higher, respectively. Meanwhile, the velocity estimation precision of three axes are improved about $19.9 \%, 13.3 \%, 17.6 \%$, respectively. As position can be obtained indirectly through velocity measurement, their improvements are fewer than velocity. Meanwhile, the inference which is proposed in the end of last section is now be proved.

\section{Conclusion}

This paper has described the INS/SAR/GPS integrated navigation system of UAVs. The RVS approach is introduced first and an optimal data fusion algorithm through RVS is applied to modified the global data fusion algorithm in integrated system. The RVS approach use more information sources than FKF to obtain the optimal data fusion, so it will achieve a higher level of accuracy with a familiar calculating time. A simulation with realistic flight data is implemented, under these circumstances, RVS approach improve the position accuracy by $1.29 \sim 1.59 \%$ and velocity accuracy by $13.3 \sim 19.9 \%$. Thus, applied in global fusion process, RVS approach is superior to FKF in estimation accuracy.

\section{Reference}

1. Rigatos, Gerasimos G. Distributed filtering over sensor networks for autonomous navigation of UAVs, Intelligent Service Robotics, 5.3.179-198 (2012)

2. Hannah P. Starr, A Decision in condition monitoring-An example for data fusion architecture, Proceedings of 2000 International Conference on Information Fusion, France, Paris, 291-298(2000)

3. Olfati-Saber, Reza, Distributed Kalman filtering for sensor networks, 46th IEEE Conference on IEEE Decision and Control (2007)

4. N. A. Carlson, Federated filter for fault-tolerant integrated navigation systems, Proceedings of IEEE Position Location and Navigation Symposium, 110-119(1988)

5. Gu Yuntao, Research on the navigation technology of UAVs, Modern Navigation, 198-202(2013)

6. Gao, Shesheng, et al, Multi-sensor optimal data fusion for INS/GPS/SAR integrated navigation system, Aerospace Science and Technology, 13.4.232-237(2009)

7. David H. Titterton, Strapdown Inertial Navigation Technology, The Institution of Electrical Engineer, 15-22(2010).

8. Dan Simon, Optimal State Estimation: Kalman, H Infinity, and Nonlinear Approaches, John Wiley\&Sons Limited,88-95(2006)

9. Mu Rongjun, Multisource information fusion and fault tolerant technology of RLV, Harbin Institute of Technology, China, 100-115(2006)

10. N. A. Carlson, Federated filter or computer-efficient, near-optimal GPS integration, Proc.IEEE Position Location Navigat. Symp.(PLANS), 96.306-314(1996)

11. Y. Li, Decentralized filtering theory and its application to the spacecraft navigation using GPS, Ph.D. Dissertation, Northwestern Polytechnic University, China(1997)

12. C. Gauss, Theory of Motion of the Heavenly Bodies Moving About the Sun in Conical Sections: A Translation of Theoria Motus, Dover, New York(2004)

13. W. Jiang, Y. L, Chris Rizos, Optimal Data Fusion Algorithm for navigation using triple integration of PPP_GNSS, INS and Terrestrial Ranging System, IEEE Sensors Journal, 15. 5634-5644(2015)

14. Y. Li, Optimal multisensory integrated navigation through information space approach, Physical Communication,13.44-53(2014) 